\title{
Energetic Cost for Being "Redox-Site-Rich" in Pseudocapacitive Energy Storage with Nickel - Aluminum Layered Double Hydroxide Materials
}

Xianghui Zhang a, b, Cody B. Cockreham ${ }^{\text {a, b, c }}$, Esra Y1lmaz ${ }^{\text {a, b, d }}$, Gengnan Li ${ }^{\text {a, b }}$, Nan Li e, Su Ha

, , Liangjie Fu ${ }^{\mathrm{f}}$, Jianqi Qi ${ }^{\mathrm{g}}$, Hongwu Xu ${ }^{\mathrm{c}}$, and $\mathrm{Di} \mathrm{Wu}^{\mathrm{a}, \mathrm{b}, \mathrm{h}, \mathrm{i}, *}$

a Alexandra Navrotsky Institute for Experimental Thermodynamics, Washington State

University, Pullman, Washington 99163, United States

${ }^{\mathrm{b}}$ The Gene and Linda Voiland School of Chemical Engineering and Bioengineering, Washington State University, Pullman, Washington 99163, United States

${ }^{\mathrm{c}}$ Earth and Environmental Sciences Division, Los Alamos National Laboratory, Los Alamos, New Mexico 87545, United States

${ }^{\mathrm{d}}$ Department of Chemical Engineering, Ege University, Izumir 35100, Turkey

${ }^{\mathrm{e}}$ Center for Integrated Nanotechnologies, Los Alamos National Laboratory, Los Alamos, New Mexico 87545, United States

${ }^{\mathrm{f}}$ Centre for Mineral Materials, School of Minerals Processing and Bioengineering, Central South University, Changsha, Hunan 410083, China

g College of Physics, Sichuan University, Chengdu, 610064, China

${ }^{\mathrm{h}}$ Department of Chemistry, Washington State University, Pullman, Washington 99163, United States

i Materials Science and Engineering, Washington State University, Pullman, Washington 99163, United States

Corresponding Author:

Di Wu, d.wu@wsu.edu 


\section{Abstract}

Defining the energetic landscape of pseudocapacitive materials such as transition metal layered double hydroxides (LDHs) upon redox site enrichment is essential to harness their power for effective energy storage. Here, coupling acid solution calorimetry, in situ XRD, and in situ DRIFTS, we demonstrate that as the $\mathrm{Ni} / \mathrm{Al}$ ratio increases, both as-made (hydrated) and dehydrated NiAl-LDH samples are less stable evidenced by their enthalpies of formation. Moreover, the higher specific capacity at intermediate $\mathrm{Ni} / \mathrm{Al}$ ratio of 3 is enabled by effective water $-\mathrm{LDH}$ interactions, which energetically stabilizes the excessive near-surface Ni redox sites, solvates intercalated carbonate ions, and fills the expanded vdW gap, paying for the "energetic cost" of being "redox site rich". Thus, from a thermodynamic perspective, engineering molecule/solids LDH interactions on the nanoscale with confined guest species other than water, which energetically impose stronger stabilization, may help us to achieve their specific capacitance potential.
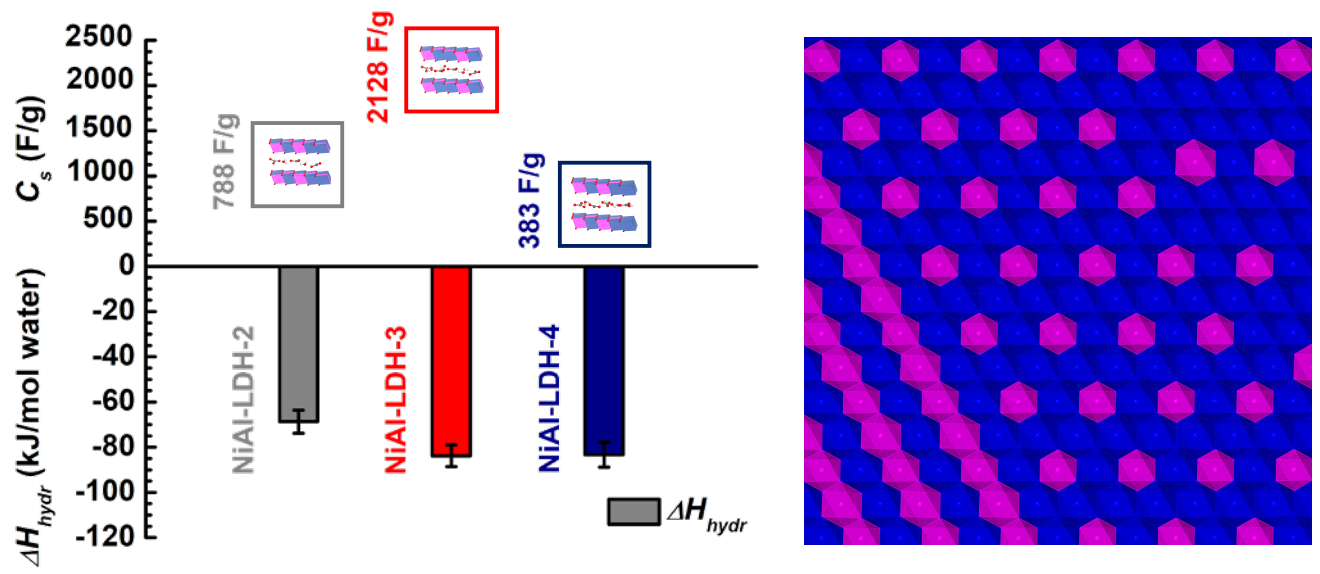


\section{Introduction}

Electrochemical energy storage devices, primarily batteries and capacitors, are playing critical roles in the ongoing modification of our energy infrastructure ${ }^{1-6}$. Rechargeable battery materials rely on phase transitions and/or solid-state reactions during the charge-discharge processes, while capacitive energy storage mechanisms take advantage of the electrostatic charge storage at or near the surface of the electrode materials ${ }^{7-10}$. Compared with the "endurance athletes" - rechargeable batteries that have high energy density, the advantages of electrochemical capacitors include high power density, long cycle life, and rapid charge - discharge. These properties enable high performance "sprinter" energy storage devices for short-term charge storage, regenerative braking, and burst-mode delivery of energy ${ }^{11-15}$.

Depending on the working mechanisms, electrochemical capacitors are typically categorized as (1) electrical double-layer capacitors (EDLCs or supercapacitor) and (2) pseudocapacitors ${ }^{11-15}$. EDLCs rely on the formation of an ionic double layer at the electrode electrolyte interfaces for electrical energy storage. Despite their low cost and wide availability, the carbon-based EDLCs suffer from low energy density because of the limitation of surface area and effective interfaces ${ }^{16-20}$. In this regard, pseudocapacitors feature substantially increased energy density accompanied with high power density, rapid charge transfer kinetics, and cycle stability ${ }^{21-26}$. Fundamentally, pseudocapacitive materials store charge at or near their surfaces by reversible redox reactions based on Faradaic charge transfer, in which the concentration of redox sites are a governing factor of the specific capacitance ${ }^{22,27,28}$. Thus, understanding the impacts of redox site concentration on the phase stability, structure, and storage of pseudocapacitive materials, such as transition metal oxides ${ }^{22,25,26}$, hydroxides ${ }^{12,13}$, and sulfides ${ }^{29,30}$ along with conductive polymers ${ }^{31}$, is essential for further materials discovery, rational design, and optimization. 
This paper elucidates the energetics - structure - performance relationships of transition metal layered double hydroxides (TM-LDHs) as the redox site concentration is tuned. TM-LDHs possess positively charged brucite-like metal hydroxide layers constructed by $\mathrm{M}^{2+}$ and $\mathrm{M}^{3+}$ transition metal octahedra ${ }^{32}$. Introduction of trivalent cations $\mathrm{M}^{3+}$ into the $\mathrm{M}^{2+}\left(\mathrm{OH}^{-}\right)_{2}$ layer leads to layer-possessed positive charges and simultaneous intercalation of charge-balancing anions $\left(\mathrm{A}^{\mathrm{n}}\right)$ confined within the interlayer space, which is also filled by neutrally charged guest species, such as water molecules ${ }^{12,33}$. The open 2-dimensional structure of TM-LDHs enables highly efficient Faradic redox reactions at the near-surface transition metal sites with fast kinetics. In an earlier study of NiAl-LDH with $\mathrm{CO}_{3}{ }^{2-}$ as the charge-balancing anions, we demonstrate that an increase in $\mathrm{Ni} / \mathrm{Al}$ ratio from 2 to 4 leads to increased $\mathrm{Ni}$ redox site concentration, expanded van der Waals (vdW) gap resulting in faster charge transport kinetics, and degraded near-surface crystallinity ${ }^{21}$. We also find that the pseudocapacitive charge storage capacity is not positively correlated to the $\mathrm{Ni} / \mathrm{Al}$ ratio. Additionally, high near-surface crystallinity is crucial to ensure high pseudocapacitive capacitance ${ }^{21,22,25}$. However, the thermodynamic mechanisms for the complex interplay among Ni redox site concentration, near-surface crystallinity, and intercalation chemistry of NiAl-LDHs have not been clearly and quantitatively identified from microscopic and macroscopic perspectives.

Here, we report a systematic study that elucidates the impacts of Ni content on the energetic stability and thermodynamics - structure - performance relationship of NiAl-LDH by integrating two powerful in situ techniques, in situ X-ray diffraction (XRD) and in situ diffuse reflectance infrared Fourier transform spectroscopy (DRIFTS), with the unique calorimetric methods in our lab, including near-room temperature acid solution calorimetry. The obtained results suggest that Ni enrichment leads to decreased energetic, thermal, structural, 

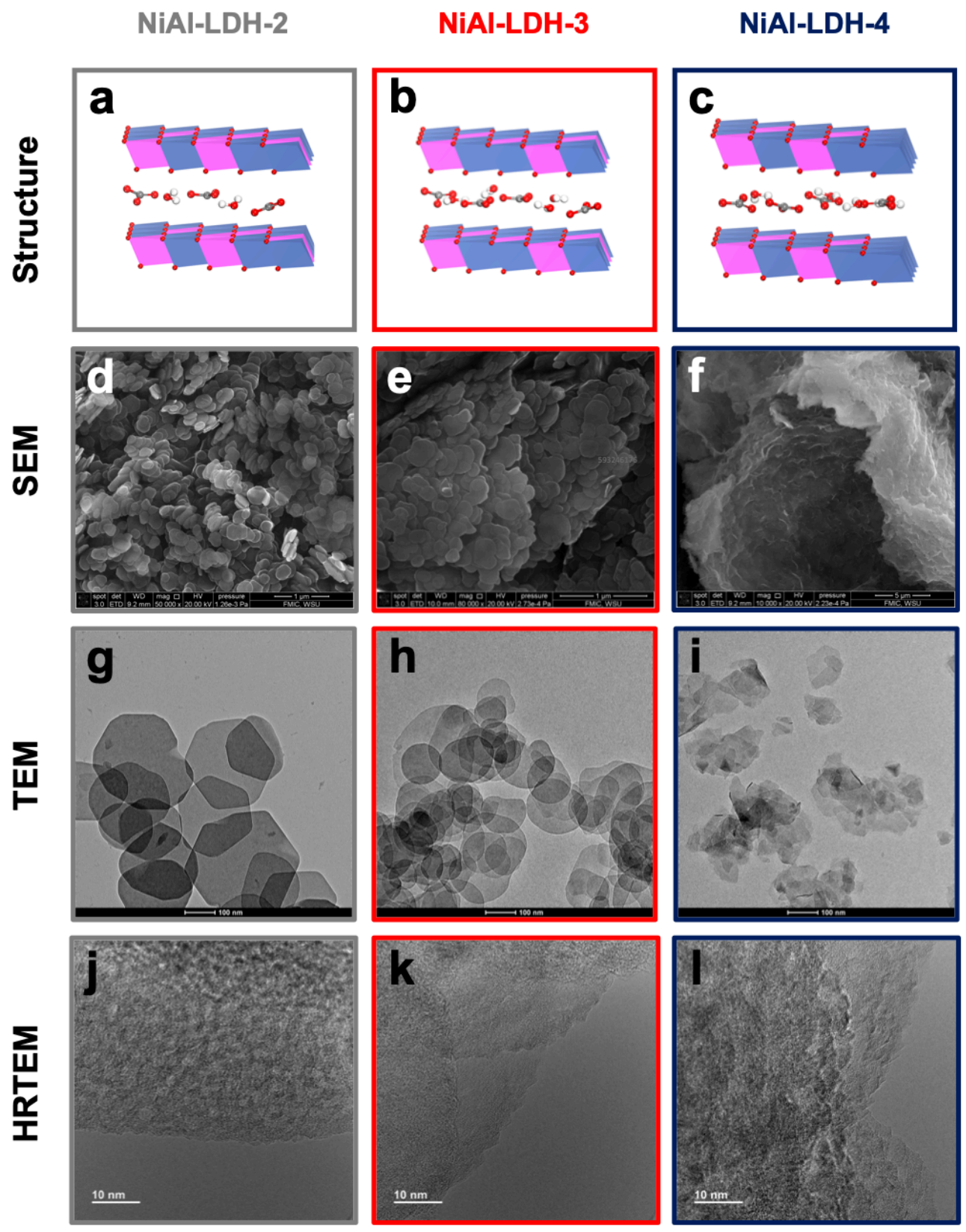

Figure 1. Illustrated structures $(\mathbf{a}, \mathbf{b}, \mathbf{c})$ and morphologies of NiAl-LDH samples revealed by scanning electron microscopy (SEM, d, e, f), transmission electron microscopy (TEM, g, h, i), and high-resolution transmission electron microscopy (HRTEM, j, k, l), NiAl-LDH-2 (gray), NiAlLDH-3 (red), and NiAl-LDH-4 (navy). 
and morphological stability for both as-made (hydrated) and dehydrated NiAl-LDHs. Further, we demonstrate that the intercalated (2D-confined) water molecules substantially neutralize the significant metastability introduced by Ni redox site enrichment, and the optimized specific capacitance is the result of the subtle balance between the energetic stability of LDH layers and water - layer guest - host interactions. This work also highlights the power of integrating in situ structural, spectroscopy, and thermodynamic techniques to unravel fundamental mechanisms underlying the properties and behavior of energy storage materials at application conditions.

\section{Results and Discussion}

\section{Composition, Morphology, Structure, and Surface Analyses}

The compositions of all samples are listed in Table S1. The illustrated structures and electron microscopy images are presented in Figure 1. As seen from the SEM and TEM images, NiAl-LDH-2 possesses hexagonal platelet nanosheet morphology, while the shape of NiAl-LDH3 and 4 platelet nanosheets appear to lack well-defined geometry. Investigated by HRTEM, NiAlLDH-4 appears nearly amorphous at atomic scales in the interplanar direction with many jagged grain boundary defects near the layer-edges. By contrast, NiAl-LDH-2 and NiAl-LDH-3 appear to have better near-surface crystallinity than NiAl-LDH-4 with smooth rounded layer-edges. As the Ni/Al ratio increases, the particle size decreases from $\sim 250 \mathrm{~nm}$ for NiAl-LDH-2 to $\sim 80 \mathrm{~nm}$ for NiAl-LDH-4. The $\mathrm{N}_{2}$ adsorption and desorption full isotherm analysis data are plotted in Figure $\mathbf{2}$ and summarized in Table $\mathbf{S 1}$. The specific surface area increases as the Ni/Al ratio increases. Specifically, the BET specific areas are 47.5, 77.0, and $152.1 \mathrm{~m}^{2} / \mathrm{g}$ for NiAl-LDH-2, 3, and 4, respectively. This trend is consistent with the particle size decrease observed in the electron microscopy analyses. In addition, in BJH pore size analysis, NiAl-LDH-3 presents the largest pore size of $22.3 \mathrm{~nm}$, while NiAl-LDH-4 has the smallest mesoscale porosity of $8.0 \mathrm{~nm}$. The pore 
a

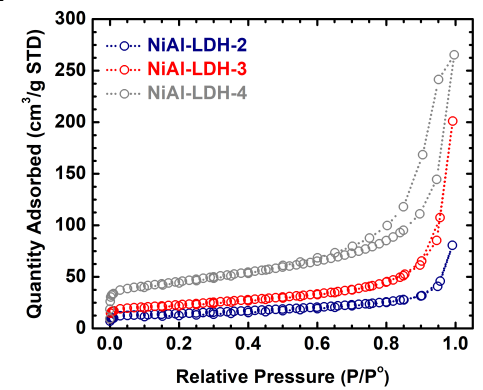

C

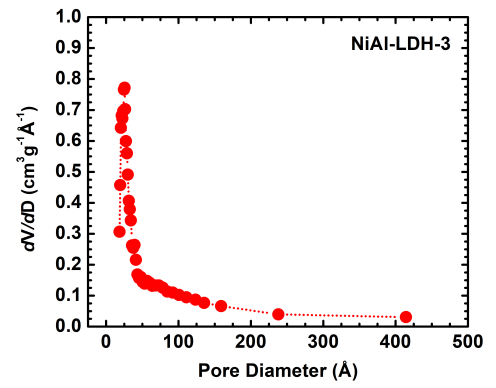

b

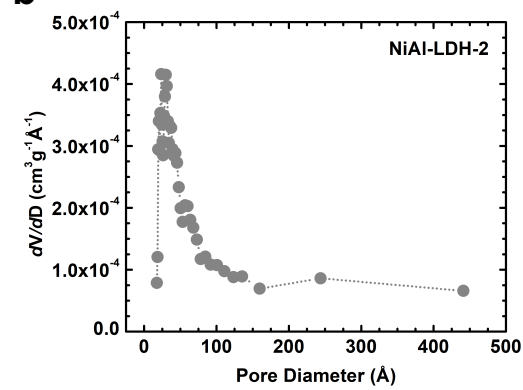

d

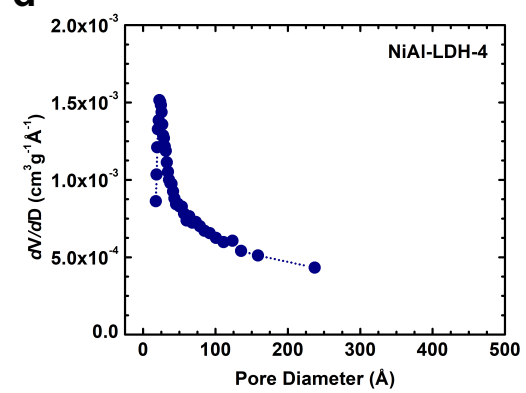

Figure 2. (a) Nitrogen adsorption and desorption full isotherms and pore size distribution of (b) NiAl-LDH-2, (c) NiAl-LDH-3, and (d) NiAl-LDH-4.

a

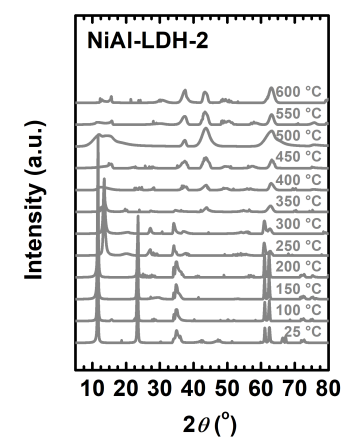

b

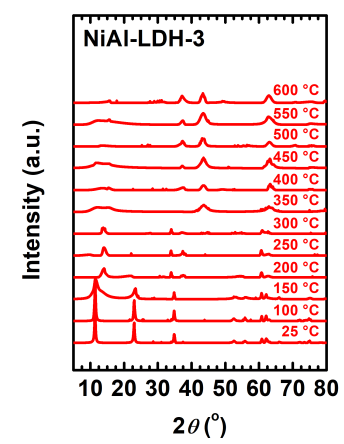

C

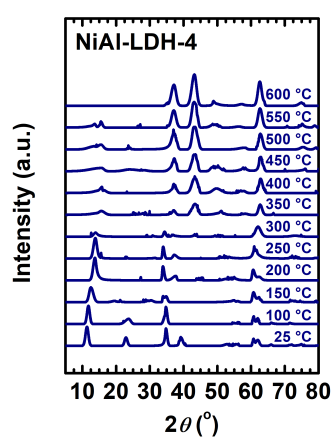

d

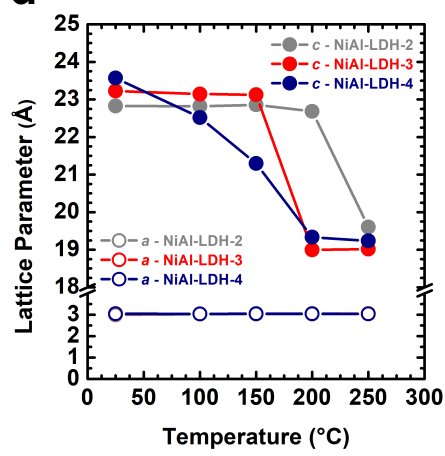

Figure 3. In Situ X-ray diffraction (XRD) patterns of all samples as a function of temperature from 25 to $600{ }^{\circ} \mathrm{C}$ at $10{ }^{\circ} \mathrm{C} / \mathrm{min}$ under helium flow of $30 \mathrm{ml} / \mathrm{min}$ with a hold time of 5 minutes at each temperature prior to measurement. Data were collected from 5 to $80^{\circ}$. (a) NiAl-LDH-2, (b) NiAlLDH-3, and (c) NiAl-LDH-4. (d) Lattice parameters of all NiAl-LDH samples as a function of temperature. Note, the $a$ parameters of all three NiAl-LDH samples overlap each other due to the similarity in their intraplanar crystallinity. 
dimension of NiAl-LDH-2 is $15.8 \mathrm{~nm}$. In general, Ni enrichment leads to decreased particle size, degraded morphology, increased surface area, and decreased pore size.

\section{Structural Evolutions}

In situ XRD patterns for NiAl-LDH samples are presented in Figure 3a, b, and $\mathbf{c}$. All samples exhibit hydrotalcite-like structures representative of metal hydroxide layered structures (PDF\#15-0087, also see Figure S1). Generally, before eventual degradation as the temperature increases, the representative XRD peaks of each NiAl-LDH broaden and shift to higher $2 \theta \mathrm{s}$. Phase transition due to degradation of the brucite-like metal hydroxide layered structure was observed at above $300{ }^{\circ} \mathrm{C}$ for NiAl-LDH-2 and 3, and $250{ }^{\circ} \mathrm{C}$ for NiAl-LDH-4. At temperatures higher than $400{ }^{\circ} \mathrm{C}$, each sample decomposes to a mixed phase of $\mathrm{NiO}$ and $\mathrm{Al}_{2} \mathrm{O}_{3}$, shown in Figure S2. Lattice parameters of the NiAl-LDHs in hexagonal close packing found from representative peaks of (003) and (110) are presented as a function of temperature in Figure 3d. The $a$ lattice parameter, which represents the cell dimension parallel to the brucite-like layer, does not change appreciably throughout the temperature range investigated. Seen in our previous work and repeated here, the $c$ lattice parameter (related to the $d$-spacing by the equation $c=3 \times d_{003}$ ) at $25^{\circ} \mathrm{C}$, which represents the cell dimension in the interlayer direction, increases with $\mathrm{Ni}$ content ${ }^{21}$. As Ni content increases and Al content decreases, the total positive charge of the metal hydroxide layer lessens; proportionately, interlayer charge balancing anions decrease limiting electrostatic interactions ${ }^{21}$. As temperature increases, the $c$ lattice parameter of each sample decreases. The $c$ lattice parameters exhibit $3 \sim 4 \AA$ relatively abrupt decrease from 200 to $250{ }^{\circ} \mathrm{C}$ for NiAl-LDH-2, and from 150 to $200{ }^{\circ} \mathrm{C}$ for NiAl-LDH-3. Such stepwise decrease is indicative of the loss of one layer of intercalated water (dehydration), since interlayer-confined water molecules are $\sim 2.5 \AA$ in size ${ }^{34}$. Interestingly, NiAl-LDH-3 seemingly expands its interlayer distance from 250 to $300{ }^{\circ} \mathrm{C}$. We 
attribute this to be normal lattice thermal expansion or an artifact from the growing disorder in stacking, while carbonate deintercalates and subsequent structural degradation observed from the wide distribution of the (003) peak at $300{ }^{\circ} \mathrm{C}$ (see Figure 3b). The $c$ parameter of NiAl-LDH-4 features a much more gradual decrease for $\sim 4 \AA$ spanning from 25 to $200{ }^{\circ} \mathrm{C}$, presumably due to more chaotic stacking (seen in SEM and/or TEM) leading to varied interlayer environments. As Ni content increases interlayer dehydration occurs at lower temperatures. In summary, the in situ XRD results strongly highlights (1) the loss of interlayer space after dehydration of intercalated water, and (2) phase transition to oxide phases at temperature above $400{ }^{\circ} \mathrm{C}$, which is accompanied by release of $\mathrm{CO}_{2}$ (see the TG-DSC-MS data plotted in Figure S3) ${ }^{21}$.

\section{Interfacial Phenomena}

The in situ DRIFTS spectra suggest all NiAl-LDH samples share three vibrational features (see Figure 4 and S3), including (1) a broad absorption spanning from 4000 to $2500 \mathrm{~cm}^{-1}$ owing to the $\mathrm{O}-\mathrm{H}$ stretching vibration of hydroxyl groups from the brucite-like layers and intercalated water molecules; (2) three overlapped peaks between 2000 and $650 \mathrm{~cm}^{-1}$ due to interlayer-confined $\mathrm{H}_{2} \mathrm{O}$ and carbonate species, and surface $\mathrm{OH}$ groups, specifically, one at $\sim 1650 \mathrm{~cm}^{-1}$ (bending vibration of adsorbed water), and two between 1370 and $1000 \mathrm{~cm}^{-1}$ (asymmetric stretching of interlayer carbonate species); (3) several relatively weak absorption peaks between 1000 and 650 $\mathrm{cm}^{-1}$ belonging to lattice vibration of the metal - oxygen bonds within the NiAl-LDH layer.

Both in situ DRIFTS and TG-DSC-MS results strongly suggest a two-stage mechanism for thermal degradation of NiAl-LDH. Specifically, in Stage I interlayer dehydration (from 25 to 200 $\left.{ }^{\circ} \mathrm{C}\right)$, starting from $\sim 150{ }^{\circ} \mathrm{C}$ a sharp peak at $3680 \mathrm{~cm}^{-1}$ appears, which is assigned to $v(\mathrm{OH})$ of isolated surface hydroxyl groups of $\mathrm{LDH}^{35}$. Interestingly, although this peak is clearly well- 

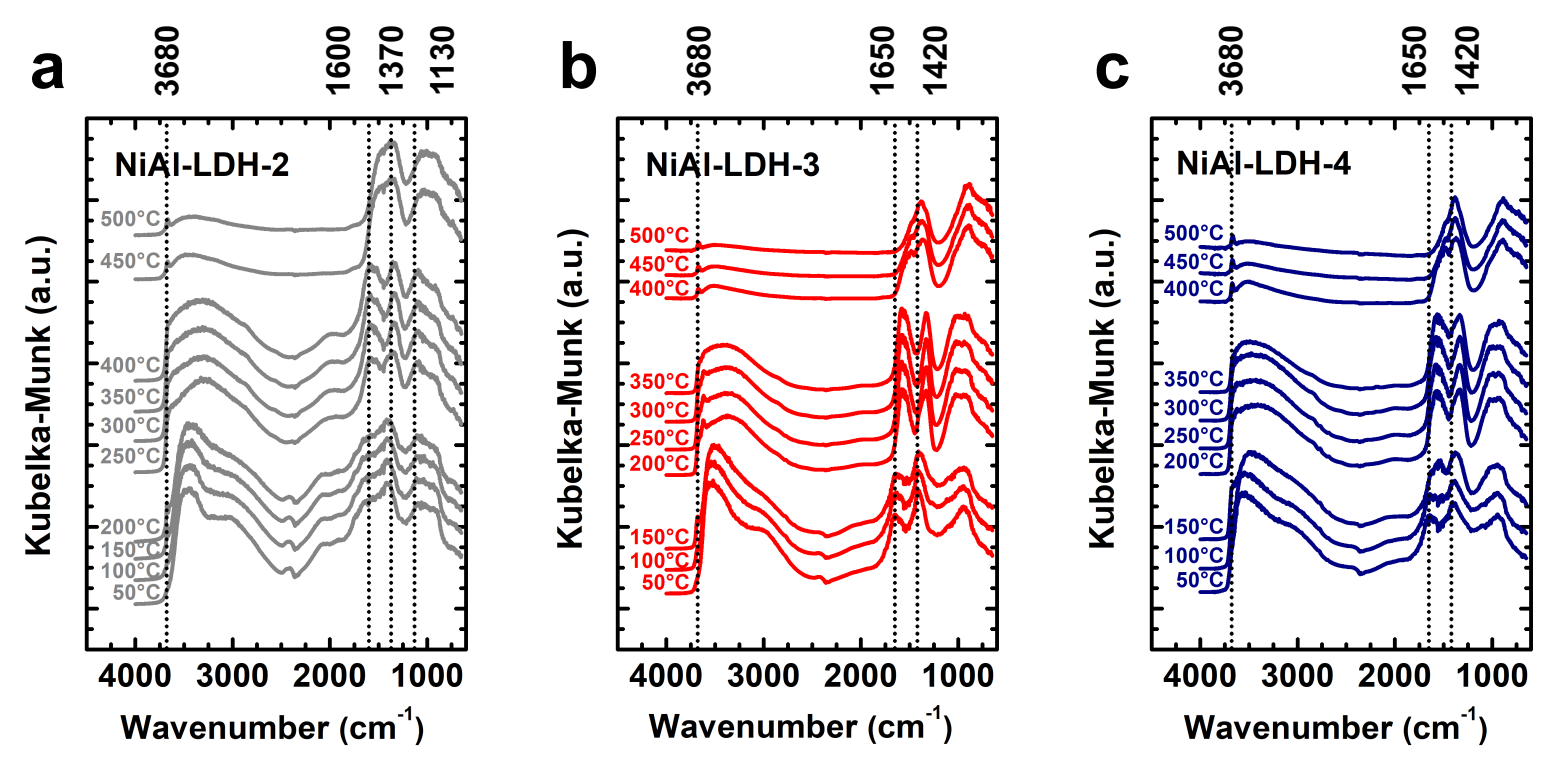

Figure 4. In Situ diffuse reflectance infrared Fourier transform spectroscopy (DRIFTS) spectra of each sample as a function of temperature from 50 to $500{ }^{\circ} \mathrm{C}$ under helium flow of $50 \mathrm{ml} / \mathrm{min}$, (a) NiAl-LDH-2, (b) NiAl-LDH-3 and (c) NiAl-LDH-4.

resolved for NiAl-LDH-2 and 3, it appears to be broad and nearly undetectable during Stage I dehydration of NiAl-LDH-4. Dehydration in Stage I leads to removal of intercalated water molecules reducing the hydrogen bonding among interlayer adsorbed/confined species. As temperature increases, the low wavenumber peaks of NiAl-LDH-2 become better resolved, splitting into three sharp peaks centered at 1600, 1370, and $1130 \mathrm{~cm}^{-1}$. For NiAl-LDH-3 and 4, shift from 1650 to $1600 \mathrm{~cm}^{-1}$ and from 1420 to $1370 \mathrm{~cm}^{-1}$ were observed. The signal at $\sim 1600 \mathrm{~cm}^{-}$ ${ }^{1}$ is attributed to the bending mode of exposed surface $\mathrm{OH}$ groups from $\mathrm{Ni}(\mathrm{OH})_{2}$ and $\mathrm{Al}(\mathrm{OH})_{3}{ }^{36}$. Hence, right-shift from 1650 to $1600 \mathrm{~cm}^{-1}$ is caused by dehydration of layer-confined water molecules. Right-shift of the peak at $1420 \mathrm{~cm}^{-1}$ due to $v(\mathrm{C}-\mathrm{O})$ of carbonate anions arises from significantly enhanced anion - layer interactions after dehydration ${ }^{35}$. Interestingly, all post Stage 
I dehydration samples present similar DRIFTS spectra. This is because removal of water molecules from the interlayer space significantly reduces the chemical complexity of guest - host interactions among various intercalated (confined) species (mainly carbonates) and the NiAl-LDH layers. After complete interlayer dehydration, the high wavenumber broad peak spanning from 4000 to $2500 \mathrm{~cm}^{-1}$ becomes near-absent. The remaining peaks are mainly from the layer-possessed functional groups and charge-balancing carbonates.

Stage II degradation of the LDH structure from $\sim 200{ }^{\circ} \mathrm{C}$ concludes at $\sim 450{ }^{\circ} \mathrm{C}$ for NiAlLDH-2 and at about $400{ }^{\circ} \mathrm{C}$ for NiAl-LDH-3 and 4, in which two significant transitions are detected by in situ DRIFTS. Specifically, the intensity of signal at $1370 \mathrm{~cm}^{-1}$ decreases as temperature increases, which strongly suggests decomposition of interlayer confined carbonate species to $\mathrm{CO}_{2}$ that was also seen within the same temperature range in the TG-DSC-MS thermal analysis ${ }^{33,34}$. Meanwhile, the signal at $\sim 1600 \mathrm{~cm}^{-1}$ completely disappears. According to the in situ XRD data, this is caused by thermal decomposition of $\mathrm{Ni}(\mathrm{OH})_{2}$ and $\mathrm{Al}(\mathrm{OH})_{3}$, and phase transition to $\mathrm{NiO}$ and $\mathrm{Al}_{2} \mathrm{O}_{3}$ (see Figure 3 and Figure S2). This set of in situ DRIFTS data is in excellent agreement with TG-DSC-MS and in situ XRD results, which reveal a two-stage degradation mechanism, including Stage I interlayer dehydration leading to the loss of LDH's interlayer gallery; and Stage II degradation of LDH layers with significant phase transition of all NiAl-LDH samples induced by the decomposition of the anionic charge-balancing carbonate species.

\section{Energetics of As-made and Dehydrated NiAl-LDHs}

According to the TG-DSC-MS thermal analysis results, the integral enthalpies of hydration $\left(4 H_{h y d r}\right)$ of NiAl-LDH samples are $-68.68 \pm 5.12,-83.80 \pm 4.81$ and $-83.32 \pm 5.53 \mathrm{~kJ} / \mathrm{mol}$ water, for NiAl-LDH-2, 3, and 4, respectively. The directly measured enthalpies of dissolution of all 


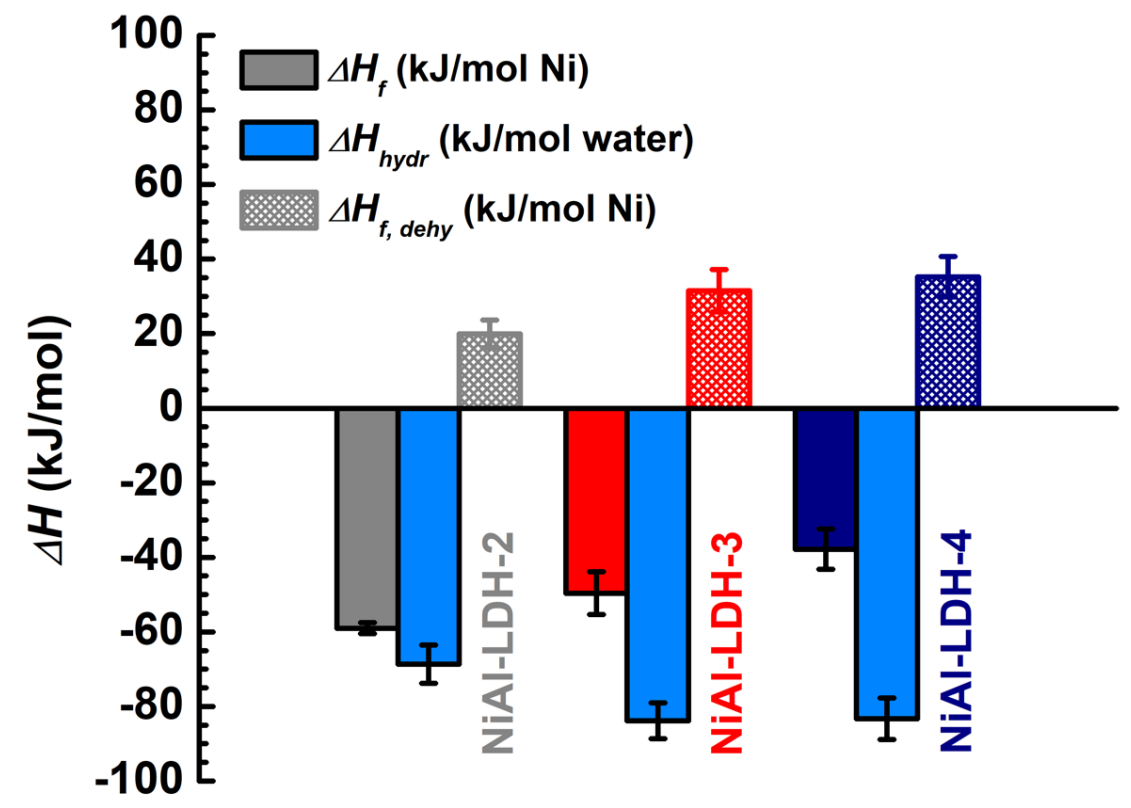

Figure 5. Enthalpies of formation from $\mathrm{NiO}(\mathrm{s}), \mathrm{Al}(\mathrm{OH})_{3}(\mathrm{~s}), \mathrm{H}_{2} \mathrm{O}(\mathrm{l})$, and $\mathrm{CO}_{2}(\mathrm{~g})$ at $25{ }^{\circ} \mathrm{C}$ for all NiAl-LDH samples.

a

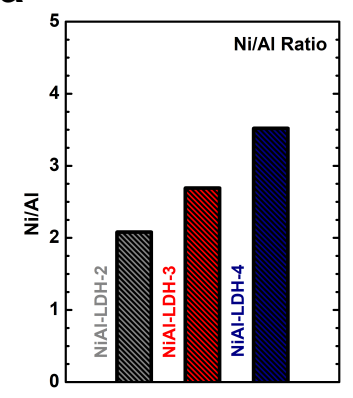

b

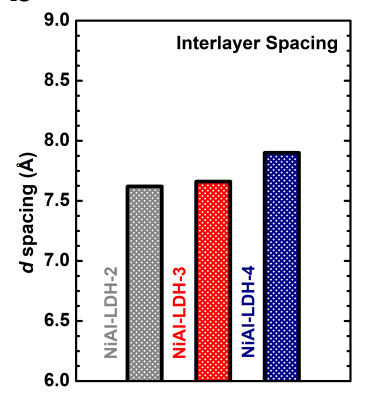

c

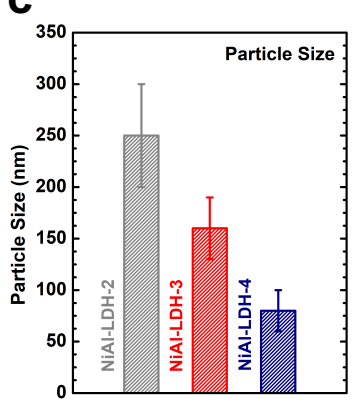

d

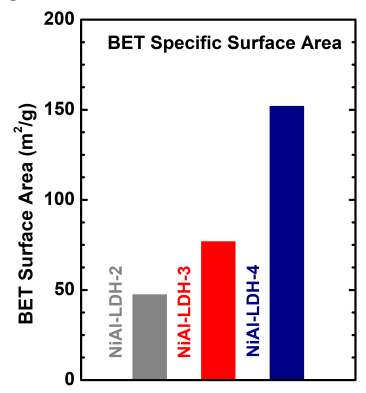

Figure 6. (a) Ni/Al ratio, (b) $d$-spacing reflecting interlayer spacing, (c) particle size for all NiAlLDH samples, and (d) Brunauer-Emmett-Teller (BET) specific areas ${ }^{21}$. 
samples in $\mathrm{HCl}$ aqueous solution $(5 \mathrm{~N})$ at $40^{\circ} \mathrm{C}$ are listed in Table S2. The enthalpies of formation, $\Delta H_{f}$, for NiAl-LDH samples from $\mathrm{NiO}, \gamma-\mathrm{Al}(\mathrm{OH})_{3}, \mathrm{H}_{2} \mathrm{O}$, and $\mathrm{CO}_{2}$, are calculated using the thermodynamic cycle listed in Table S3. In general, formation of the as-made fully hydrated NiAlLDHs is energetically favorable, reflected by the negative formation enthalpy $\left(\Delta H_{f}\right)$ values. $\Delta H_{f}$ tends to be less exothermic as the $\mathrm{Ni} / \mathrm{Al}$ ratio or interlayer spacing increases, ranging from -59.00 \pm 1.46 to $-37.80 \pm 5.37 \mathrm{~kJ} / \mathrm{mol} \mathrm{Ni}$ (see Figure 5). Further, with the enthalpies of formation and dehydration, we are able to calculate the formation enthalpies of dehydrated NiAl-LDH samples with the interlayer confined water removed, $\Delta H_{f-d e h y}$. Surprisingly, all $\Delta H_{f-d e h y}$ values are positive, strongly suggesting the dehydrated NiAl-LDH samples are energetically unfavorable. Moreover, $\Delta H_{f-\text { dehy }}$ becomes more endothermic as the $\mathrm{Ni} / \mathrm{Al}$ ratio increases. Specifically, $\Delta H_{f-\text { dehy }}$ are $19.95 \pm$ $6.06,31.50 \pm 7.36$, and $35.26 \pm 7.24 \mathrm{~kJ} / \mathrm{mol} \mathrm{Ni}$ for NiAl-LDH 2, 3, and 4, respectively (see Figure 5). Table S4 lists all calorimetric data discussed above. All data are presented with error bars.

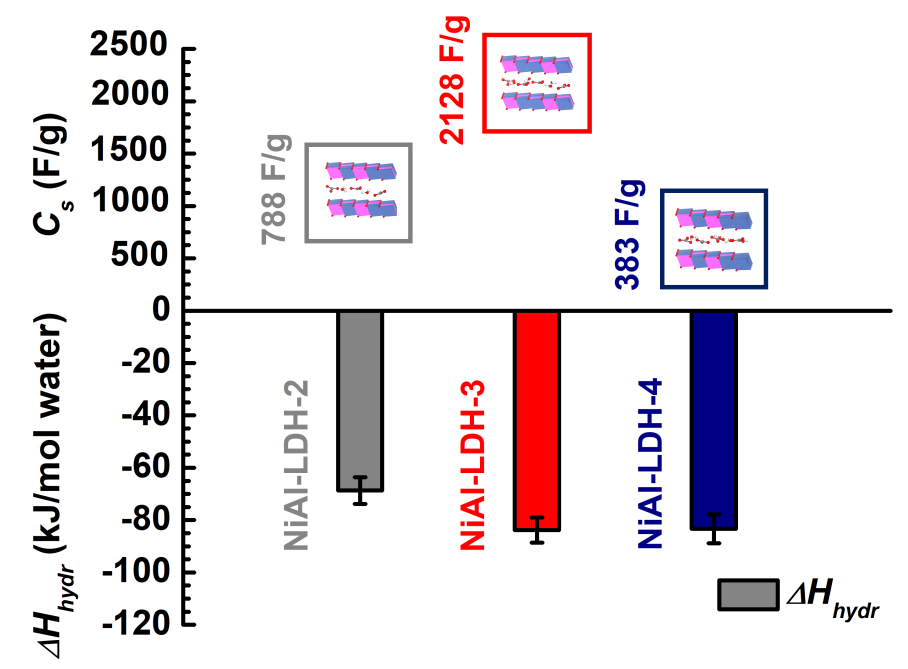

Figure 7. Energetic - structure - performance relationships for all NiAl-LDHs with Ni/Al from 2 to 4 . The specific capacity $\left(C_{s}\right)$ values were cited from reference ${ }^{21}$. 


\section{Energetic Landscape of NiAl-LDH, Critical Role of Water Intercalation, and Structure - Energetics - Performance Relationships}

Our earlier study demonstrated that increase in $\mathrm{Ni} / \mathrm{Al}$ ratio leads to (1) increase of $\mathrm{Ni}$ redox site concentration, (2) expansion of interlayer space (van der Waals gap), (3) degradation of crystallinity and morphology (see Figure 1 and 3), (4) enhancement of ion transport kinetics, and (5) modification of specific capacitance (F/g) with the highest capacity recorded for NiAl-LDH-3 33. All these trends are summarized in Figure 6. Using in situ methodologies and from a thermodynamic perspective, our present study leads to the following general conclusions: first, as the Ni content increases, both as-made (hydrated) and dehydrated NiAl-LDHs tend to be energetically less stable, and all dehydrated NiAl-LDH samples are no longer stable, reflected by the positive formation enthalpies $\left(\Delta H_{f-d e h y}\right)$. Although decreased stability upon introduction of more redox defects is a common phenomenon seen for solid state oxide and hydroxide materials ${ }^{37-39}$, the endothermic enthalpies of formation for dehydrated NiAl-LDH are not typically seen, which indicates that upon removal of the intercalated water the NiAl-LDH is unstable and will readily transform to more energetically favorable phases. Hence, interlayer-confined species such as small molecules, ions, and/or materials are essential to minimize or neutralize the metastability generated by redox site enrichment. In other words, interlayer-confined species pay the "cost of being redox site rich". Second, hydration plays critical thermodynamic roles in determination of the functionality of NiAl-LDH structures by (i) energetically maintaining the open interlayer gallery structure for electrolyte intercalation with rapid kinetics; (ii) stabilizing the interlayerconfined charge-balancing carbonates by acting as solvents; and (iii) minimizing the metastability generated by highly concentrated layer-possessed hydroxyls in NiAl-LDHs. The energetic balance through subtle interplay between compositional factors and water - layer guest - host interactions 
govern the specific capacitance of NiAl-LDH materials (see Figure 7). Lastly, inspired by the thermodynamic insights into NiAl-LDH formation and hydration obtained here, we anticipate that we will be able to pay the "energetic cost of being redox site rich" by interfacial engineering strategies to tune the chemical nature and magnitudes of guest - host interactions to synthesize pseudocapacitive energy storage materials with enhanced stability, faster kinetics, and higher capacitance. Also, we expect that by tuning the degree of hydration we may manipulate the kinetics, energetics, and capacitance of TM-LDH materials to engineer desired qualities.

In our earlier study, we have shown that $\mathrm{Ni}$ atom enrichment for NiAl-LDH leads to expansion in interlayer spacing facilitating faster kinetics and increased number of energy storage sites during the reversible charge - discharge process. Here, from a thermodynamic perspective, we further the fundamental understanding of the NiAl-LDH system by demonstrating that the increase in $\mathrm{Ni}$ redox site concentration results in decreased energetic stability for both as-made (hydrated) and dehydrated NiAl-LDHs, reflected by their enthalpies of formation. Moreover, interlayer hydration significantly stabilizes the metastability introduced by Ni enrichment. The highest specific capacity observed for NiAl-LDH-3 (2128 F/g at $1 \mathrm{~A} / \mathrm{g})$ with an intermediate Ni content is achieved by effective stabilization from hydration through subtly balanced interlayer confinement (intercalation) and interfacial binding, which intrinsically pays the "energetic cost" generated by the increase of the $\mathrm{Ni} / \mathrm{Al}$ ratio. Therefore, our study highlights the critical role of hydration, and demonstrates that interfacial engineering on the nanoscale within the interlayer space is a promising strategy to finetune the confinement chemistry and surface redox sites for substantial enhancement of the energy storage properties of transition metal layered double hydroxides (TM-LDHs). 


\section{Experimental Methods}

\section{Materials Synthesis}

All chemicals used were Sigma-Aldrich ACS reagent grade. NiAl-LDH samples were prepared hydrothermally using a Teflon-lined autoclave. Each sample was denoted as NiAl-LDH$n$ according to the $\mathrm{Ni} / \mathrm{Al}$ ratio $(n)$. Specifically, $\mathrm{Ni}\left(\mathrm{NO}_{3}\right)_{2} \cdot 6 \mathrm{H}_{2} \mathrm{O}$ (purity $>98.5 \%$ ) and $\mathrm{Al}\left(\mathrm{NO}_{3}\right)_{3} \cdot 9 \mathrm{H}_{2} \mathrm{O}$ (purity $\left.>98 \%\right)(\mathrm{Ni} / \mathrm{Al}=n$, and $\mathrm{Ni}+\mathrm{Al}=1.875 \mathrm{mmol})$ were dissolved in $25 \mathrm{ml}$ nanopure water $(18.2 \mathrm{M} \Omega \cdot \mathrm{cm})$. For $\mathrm{NiAl}-\mathrm{LDH}-2, \mathrm{Ni}\left(\mathrm{NO}_{3}\right)_{2} \cdot 6 \mathrm{H}_{2} \mathrm{O}$ (purity $>98.5 \%$ ) and $\mathrm{Al}\left(\mathrm{NO}_{3}\right)_{3} \cdot 9 \mathrm{H}_{2} \mathrm{O}$ (purity $>98 \%$ ) were 1.25 and $0.625 \mathrm{mmol}$, respectively. For NiAl-LDH-3, $\mathrm{Ni}\left(\mathrm{NO}_{3}\right)_{2} \cdot 6 \mathrm{H}_{2} \mathrm{O}$ (purity $>98.5 \%$ ) and $\mathrm{Al}\left(\mathrm{NO}_{3}\right)_{3} \cdot 9 \mathrm{H}_{2} \mathrm{O}$ (purity $>98 \%$ ) were 1.460 and 0.456 mmol, respectively. For NiAl-LDH-4, $\mathrm{Ni}\left(\mathrm{NO}_{3}\right)_{2} \cdot 6 \mathrm{H}_{2} \mathrm{O}$ (purity $>98.5 \%$ ) and $\mathrm{Al}\left(\mathrm{NO}_{3}\right)_{3} \cdot 9 \mathrm{H}_{2} \mathrm{O}$ (purity $>98 \%$ ) were 1.25 and $0.365 \mathrm{mmol}$, respectively. In addition, the precipitant used was hexamethylenetetramine (HMT, $2.20 \mathrm{mmol}$, purity > 99\%) for NiAl-LDH-2, and urea (4.13 mmol, purity $>99 \%$ ) was applied to precipitate NiAl-LDH-3 and 4. In each synthesis, the mixture was introduced into a $50 \mathrm{~mL}$ Teflon-lined autoclave for the hydrothermal reaction at $180{ }^{\circ} \mathrm{C}$ for $72 \mathrm{~h}$. The precipitates were filtered and washed with nanopure water $(18.2 \mathrm{M} \Omega \cdot \mathrm{cm})$ followed by drying under vacuum at room temperature ${ }^{21}$.

\section{Ex Situ Powder X-ray Diffraction (XRD), Compositional and Thermal Analyses}

For phase identification, room temperature ex situ powder X-ray diffraction (XRD) patterns of the synthesized NiAl-LDH samples were collected with a Rigaku Miniflex 600 diffractometer $(40 \mathrm{kV}, 15 \mathrm{~mA}$ with $\mathrm{Cu} K \alpha$ radiation, $\lambda=0.15418 \mathrm{~nm})$. Data were recorded between 5 and $80^{\circ}$ at $1 \%$ min. Compositional and thermal analyses were performed in our earlier study ${ }^{21}$. The chemical compositions of all samples were characterized using inductively coupled plasma- 
mass spectrometry (ICP-MS, Agilent 770). The TG-DSC-MS thermal analyses were carried out on a Netzsch Instrument STA 449 F5 Jupiter coupled to a QMS 403 D Aëolos quadrupole mass spectrometer in our earlier study emphasizing energy storage performance. The accuracy of enthalpy from DSC using the STA 449 F5 Jupiter is $\pm 2 \%$ for most materials. In each measurement, about $20 \mathrm{mg}$ sample was analyzed in a platinum crucible from 30 to $1000^{\circ} \mathrm{C}$ at $10{ }^{\circ} \mathrm{C} / \mathrm{min}$ under $\mathrm{N}_{2}$ flow $(60 \mathrm{ml} / \mathrm{min})$. Evolved gases of TG-DSC experiments were identified simultaneously by the coupled MS including $\mathrm{m} / \mathrm{z}=18\left(\mathrm{H}_{2} \mathrm{O}\right)$ and $44\left(\mathrm{CO}_{2}\right)$. The DSC data were also used to calculate the dehydration enthalpy of each NiAl-LDH sample. All ex situ XRD, ICP-MS, and TG-DSC-MS data have been reported in our earlier study on the same set of NiAl-LDH samples ${ }^{21}$ (see Table S1, Figure S1, and S3).

\section{$\mathrm{N}_{2}$ Adsorption and Desorption Full Isotherm Analysis}

The Brunauer-Emmett-Teller (BET) and Barrett, Joyner, and Halenda (BJH) surface area and pore size distribution analyses were carried out using $\mathrm{N}_{2}$ adsorption and desorption full isotherm analysis at $77 \mathrm{~K}$ on a Micromeritics 3Flex gas adsorption analyzer. Before each measurement, the sample was degassed in situ at the analysis port at $250^{\circ} \mathrm{C}$ for 2 hours.

\section{Electron Microscopy (EM)}

We examined the sample morphology using scanning electron microscopy (SEM, Quanta 200F), transmission electron microscopy (TEM, FEI Tecnai T20 with a $\mathrm{LaB}_{6}$ cathode at $200 \mathrm{kV}$ ) in Franceschi Microscopy and Imaging Center at Washington State University and high-resolution transmission electron microscopy (HRTEM, FEI Titan 80-300 TM with monochromator, image aberration corrector, and a PHENIX energy dispersive X-ray spectrometer detector) at Los Alamos National Laboratory. ImageJ (NIH) was employed for micrograph analysis ${ }^{40}$. The SEM specimen 
was prepared by loading the sample on carbon tape, which was placed on an aluminum stub. The specimen for TEM and HRTEM analyses were prepared by dispersing a small amount of sample powder in ethanol using ultrasonication. Subsequently, several drops of suspension were dried on 200-mesh carbon-coated nickel grid (TEM) and a 200-mesh carbon-coated copper grid (HRTEM).

\section{In Situ X-ray Diffraction (in situ XRD)}

The structural evolution of all NiAl-LDH samples as temperature increases from room temperature to $600{ }^{\circ} \mathrm{C}$ was monitored by in situ powder X-ray diffraction (in situ XRD). The data

were obtained using a Rigaku Smartlab X-ray diffractometer with $\mathrm{Cu} K \alpha$ radiation $(\lambda=1.5406 \AA)$ coupled with a platinum strip heater operated at a scan rate of $4^{\circ} / \mathrm{min}$ in the $2 \theta$ range of $5-80^{\circ}$ with a heating rate of $10^{\circ} \mathrm{C} / \mathrm{min}$ under $30 \mathrm{ml} / \mathrm{min}$ of helium with a hold time of $5 \mathrm{~min}$ prior to each scan. $d$-spacing was calculated using Bragg's law. The lattice parameters and unit cell volume of each sample were calculated. PDF database references were used to identify the phases of hydrotalcite (naturally occurring $\mathrm{Mg}_{6} \mathrm{Al}_{2}(\mathrm{OH})_{16} \mathrm{CO}_{3} \cdot 4 \mathrm{H}_{2} \mathrm{O}$ ), PDF-Card No. 00-014-0191, NiO, PDF-Card No. 01-073-1519, and $\mathrm{Al}_{2} \mathrm{O}_{3}$, PDF-Card No. 01-073-5928.

\section{In Situ Diffuse Reflectance Infrared Fourier Transform Spectroscopy (in situ DRIFTS)}

In situ diffuse reflectance infrared Fourier transform spectroscopy (in situ DRIFTS) analysis was carried out in a high-temperature cell (Spectra-Tech ${ }^{\circledR}$ ) equipped with $\mathrm{ZnSe}$ windows (IR transparent). Prior to each analysis, the sample was finely ground and placed in a ceramic crucible. The spectra were recorded from room temperature to $500{ }^{\circ} \mathrm{C}$ under helium flow (50 $\mathrm{mL} / \mathrm{min}$ ). The background spectra were collected under the same conditions. We were not able to reach as high as $600{ }^{\circ} \mathrm{C}$ as we did in the in situ XRD experiments due to instrumental limitations.

\section{Near-Room Temperature Acid Solution Calorimetry}


Near-room temperature acid solution calorimetry using a Setaram C80 microcalorimeter with $5 \mathrm{~mol} / \mathrm{L}$ hydrochloric acid $(\mathrm{HCl})$ from Sigma Aldrich was employed to measure the enthalpies of dissolution to derive the NiAl-LDH formation enthalpies. The Setaram C80 microcalorimeter employed has a sensitivity (Joule effect at $30^{\circ} \mathrm{C}$ ) of $30 \mu \mathrm{W} / \mathrm{mW}$ with a temperature accuracy of \pm $0.1 \%$ and an enthalpy accuracy of $\pm 1 \%$. In a typical measurement, a loose, hand-pressed sample pellet $(\sim 4 \mathrm{mg})$ was dropped into the $\mathrm{HCl}$ solution $(10 \mathrm{~mL}) \mathrm{kept}$ at $40{ }^{\circ} \mathrm{C}$. The slightly elevated temperature aided rapid dissolution of NiAl-LDH pellet, which led to a calorimetric peak. Similar strategy was also applied in an earlier study on porous silica using hydrofluoric acid solution calorimetry at $50{ }^{\circ} \mathrm{C}$, in which the temperature effects were negligible ${ }^{41}$. Complete sample dissolution was confirmed by the return to a flat baseline. Integration of the area under each calorimetric peak returned the heat of dissolution (kJ) for each pellet. At least 4 successful measurements were performed on each sample to ensure reproducibility. The error bars were calculated as two standard deviations of the mean. $\mathrm{KCl}$ (NIST) dissolution was used to calibrate the microcalorimeter.

\section{Acknowledgements}

This work was supported by institutional funds from the Gene and Linda Voiland School of Chemical Engineering and Bioengineering at Washington State University. Di Wu acknowledges the fund of Alexandra Navrotsky Institute for Experimental Thermodynamics. Xianghui Zhang is supported by the Chambroad Distinguished Fellowship. Cody B. Cockreham received support from the Achievement Rewards for College Scientists (ARCS) Fellowship from the ARCS Seattle Chapter. This work was performed, in part, at the Center for Integrated Nanotechnologies, an Office of Science User Facility operated for the U.S. Department of Energy (DOE) Office of Science. Los Alamos National Laboratory, an affirmative action equal 
opportunity employer, is managed by Triad National Security, LLC for the U.S. Department of Energy’s NNSA, under contract 89233218CNA000001. 


\section{Reference}

(1) Jiang, H.; Lee, P. S.; Li, C. 3D Carbon Based Nanostructures for Advanced Supercapacitors. Energy and Environmental Science. 2013.

(2) Pushparaj, V. L.; Shaijumon, M. M.; Kumar, A.; Murugesan, S.; Ci, L.; Vajtai, R.; Linhardt, R. J.; Nalamasu, O.; Ajayan, P. M. Flexible Energy Storage Devices Based on Nanocomposite Paper. Proc. Natl. Acad. Sci. U. S. A. 2007.

(3) Wu, X. L.; Jiang, L. Y.; Cao, F. F.; Guo, Y. G.; Wan, L. J. LiFePO4 Nanoparticles Embedded in a Nanoporous Carbon Matrix: Superior Cathode Material for Electrochemical Energy-Storage Devices. Adv. Mater. 2009.

(4) Liu, R.; Sang, B. L. MnO2/Poly(3,4-Ethylenedioxythiophene) Coaxial Nanowires by OneStep Coelectrodeposition for Electrochemical Energy Storage. J. Am. Chem. Soc. 2008.

(5) Mai, L.; Tian, X.; Xu, X.; Chang, L.; Xu, L. Nanowire Electrodes for Electrochemical Energy Storage Devices. Chemical Reviews. 2014.

(6) Chen, K.; Song, S.; Liu, F.; Xue, D. Structural Design of Graphene for Use in Electrochemical Energy Storage Devices. Chem. Soc. Rev. 2015.

(7) Zhou, B.; Yin, Y. J.; Zhang, H.; Wu, P.; Cai, C. X. Iron Phosphate Nanostructures Synthesized by Microwave Method and Their Applications in Biosensing. Nanotechnology 2010, 21 (42).

(8) Armand, M.; Tarascon, J. M. Building Better Batteries. Nature. 2008.

(9) Arico, A. S.; Bruce, P.; Scrosati, B.; Tarascon, J.-M.; van Schalkwijk, W. Nanostructured Materials for Advanced Energy Conversion and Storage Devices. Nat. Mater. F. Full J. TitleNature Mater. 2005, 4 (5), 366-377.

(10) Dominko, R.; Bele, M.; Goupil, J. M.; Gaberscek, M.; Hanzel, D.; Arcon, I.; Jamnik, J. Wired Porous Cathode Materials: A Novel Concept for Synthesis of LiFePO4. Chem. Mater. 2007.

(11) Chen, H.; Hu, L.; Chen, M.; Yan, Y.; Wu, L. Nickel-Cobalt Layered Double Hydroxide Nanosheets for High-Performance Supercapacitor Electrode Materials. Adv. Funct. Mater. 2014.

(12) Zhao, J.; Chen, J.; Xu, S.; Shao, M.; Zhang, Q.; Wei, F.; Ma, J.; Wei, M.; Evans, D. G.; Duan, X. Hierarchical NiMn Layered Double Hydroxide/Carbon Nanotubes Architecture with Superb Energy Density for Flexible Supercapacitors. Adv. Funct. Mater. 2014.

(13) Zhao, M.; Zhao, Q.; Li, B.; Xue, H.; Pang, H.; Chen, C. Recent Progress in Layered Double Hydroxide Based Materials for Electrochemical Capacitors: Design, Synthesis and Performance. Nanoscale. 2017.

(14) Salunkhe, R. R.; Tang, J.; Kamachi, Y.; Nakato, T.; Kim, J. H.; Yamauchi, Y. Asymmetric Supercapacitors Using 3D Nanoporous Carbon and Cobalt Oxide Electrodes Synthesized from a Single Metal-Organic Framework. ACS Nano 2015.

(15) Moosavifard, S. E.; El-Kady, M. F.; Rahmanifar, M. S.; Kaner, R. B.; Mousavi, M. F. Designing 3D Highly Ordered Nanoporous $\mathrm{CuO}$ Electrodes for High-Performance Asymmetric Supercapacitors. ACS Appl. Mater. Interfaces 2015.

(16) John Miller, P. S. Electrochemical Capacitors for Energy Management. Science (80-. ). 2008.

(17) Burke, A. Ultracapacitors: Why, How, and Where Is the Technology. J. Power Sources 2000, 91 (1), 37-50.

(18) Giri, S.; Ghosh, D.; Das, C. K. Growth of Vertically Aligned Tunable Polyaniline on 
Graphene/ZrO 2 Nanocomposites for Supercapacitor Energy-Storage Application. Adv. Funct. Mater. 2014.

(19) Chen, L. F.; Zhang, X. D.; Liang, H. W.; Kong, M.; Guan, Q. F.; Chen, P.; Wu, Z. Y.; Yu, S. H. Synthesis of Nitrogen-Doped Porous Carbon Nanofibers as an Efficient Electrode Material for Supercapacitors. ACS Nano 2012.

(20) Zhao, L.; Fan, L. Z.; Zhou, M. Q.; Guan, H.; Qiao, S.; Antonietti, M.; Titirici, M. M. Nitrogen-Containing Hydrothermal Carbons with Superior Performance in Supercapacitors. Adv. Mater. 2010.

(21) Li, G.; Zhang, X.; Qiu, D.; Liu, Z.; Yang, C.; Cockreham, C. B.; Wang, B.; Fu, L.; Zhang, J.; Sudduth, B.; et al. Tuning Ni/Al Ratio to Enhance Pseudocapacitive Charge Storage Properties of Nickel-Aluminum Layered Double Hydroxide. Adv. Electron. Mater. 2019.

(22) Brezesinski, T.; Wang, J.; Tolbert, S. H.; Dunn, B. Ordered Mesoporous $\alpha$-MoO3 with Iso-Oriented Nanocrystalline Walls for Thin-Film Pseudocapacitors. Nat. Mater. 2010. Liu, X.; Huang, J.; Wei, X.; Yuan, C.; Liu, T.; Cao, D.; Yin, J.; Wang, G. Preparation and Electrochemical Performances of Nanostructured CoxNi1-x $(\mathrm{OH}) 2$ Composites for Supercapacitors. J. Power Sources 2013.

(24) Kong, D.; Ren, W.; Cheng, C.; Wang, Y.; Huang, Z.; Yang, H. Y. Three-Dimensional NiCo2O4@Polypyrrole Coaxial Nanowire Arrays on Carbon Textiles for HighPerformance Flexible Asymmetric Solid-State Supercapacitor. ACS Appl. Mater. Interfaces 2015.

(25) Kim, H. S.; Cook, J. B.; Lin, H.; Ko, J. S.; Tolbert, S. H.; Ozolins, V.; Dunn, B. Oxygen Vacancies Enhance Pseudocapacitive Charge Storage Properties of MoO3-X. Nat. Mater. 2017.

(26) Ling, T.; Da, P.; Zheng, X.; Ge, B.; Hu, Z.; Wu, M.; Du, X. W.; Hu, W. Bin; Jaroniec, M.; Qiao, S. Z. Atomic-Level Structure Engineering of Metal Oxides for High-Rate Oxygen Intercalation Pseudocapacitance. Sci. Adv. 2018.

(27) Shao, M.; Ning, F.; Zhao, Y.; Zhao, J.; Wei, M.; Evans, D. G.; Duan, X. Core-Shell Layered Double Hydroxide Microspheres with Tunable Interior Architecture for Supercapacitors. Chem. Mater. 2012.

(28) Li, G.; Zhang, X.; Qiu, D.; Liu, Z.; Yang, C.; Cockreham, C. B.; Wang, B.; Fu, L.; Zhang, J.; Sudduth, B.; et al. Tuning Ni/Al Ratio to Enhance Pseudocapacitive Charge Storage Properties of Nickel-Aluminum Layered Double Hydroxide. Adv. Electron. Mater. 2019. Kulkarni, P.; Nataraj, S. K.; Balakrishna, R. G.; Nagaraju, D. H.; Reddy, M. V. Nanostructured Binary and Ternary Metal Sulfides: Synthesis Methods and Their Application in Energy Conversion and Storage Devices. Journal of Materials Chemistry A. 2017.

(30) Shen, L.; Yu, L.; Wu, H. Bin; Yu, X. Y.; Zhang, X.; Lou, X. W. Formation of Nickel Cobalt Sulfide Ball-in-Ball Hollow Spheres with Enhanced Electrochemical Pseudocapacitive Properties. Nat. Commun. 2015.

(31) Bryan, A. M.; Santino, L. M.; Lu, Y.; Acharya, S.; D’Arcy, J. M. Conducting Polymers for Pseudocapacitive Energy Storage. Chemistry of Materials. 2016.

(32) Xu, H.; Zhao, Y.; Hickmott, D. D.; Lane, N. J.; Vogel, S. C.; Zhang, J.; Daemen, L. L. High-Temperature Neutron Diffraction Study of Deuterated Brucite. Phys. Chem. Miner. 2013, 40 (10), 799-810.

(33) Mishra, G.; Dash, B.; Pandey, S. Layered Double Hydroxides: A Brief Review from Fundamentals to Application as Evolving Biomaterials. Applied Clay Science. 2018. 
(34) Hensen, E. J. M.; Smit, B. Why Clays Swell. J. Phys. Chem. B 2002.

(35) Gund, G. S.; Dubal, D. P.; Jambure, S. B.; Shinde, S. S.; Lokhande, C. D. Temperature Influence on Morphological Progress of $\mathrm{Ni}(\mathrm{OH}) 2$ Thin Films and Its Subsequent Effect on Electrochemical Supercapacitive Properties. J. Mater. Chem. A 2013.

(36) Wang, L. Y.; Tong, D. S.; Zhao, L. Z.; Liu, F. G.; An, N.; Yu, W. H.; Zhou, C. H. Utilization of Alum Sludge for Producing Aluminum Hydroxide and Layered Double Hydroxide. Ceram. Int. 2014.

(37) Navrotsky, A.; Ma, C.; Lilova, K.; Birkner, N. Nanophase Transition Metal Oxides Show Large Thermodynamically Driven Shifts in Oxidation-Reduction Equilibria. Science (80-. ). 2010, 330 (6001), 199-201.

(38) Birkner, N.; Navrotsky, A. Rapidly Reversible Redox Transformation in Nanophase Manganese Oxides at Room Temperature Triggered by Changes in Hydration. Proc. Natl. Acad. Sci. U. S. A. 2014.

(39) Birkner, N.; Navrotsky, A. Thermodynamics of Manganese Oxides: Sodium, Potassium, and Calcium Birnessite and Cryptomelane. Proc. Natl. Acad. Sci. U. S. A. 2017.

(40) Schneider, C. A.; Rasband, W. S.; Eliceiri, K. W. NIH Image to ImageJ: 25 Years of Image Analysis. Nature Methods. 2012.

(41) Wu, D.; Hwang, S.-J.; Zones, S. I.; Navrotsky, A. Guest-Host Interactions of a Rigid Organic Molecule in Porous Silica Frameworks. Proc. Natl. Acad. Sci. 2014, 111 (5), $1720-1725$.

(42) Wu, L.; Li, L.; Evans, S. F.; Eskander, T. A.; Moyer, B. A.; Hu, Z.; Antonick, P. J.; Harrison, S.; Paranthaman, M. P.; Riman, R.; et al. Lithium Aluminum-Layered Double Hydroxide Chlorides (LDH): Formation Enthalpies and Energetics for Lithium Ion Capture. J. Am. Ceram. Soc. 2019. 